\title{
Suicide "contagion": what we know and what we need to find out
}

\author{
India Bohanna PhD
}

See related research article by Swanson and Colman on page 870 and at www.cmaj.ca/lookup/doi/10.1503/cmaj.121377

$\mathrm{S}$ uicide is a global public health concern, claiming more than 1 million lives each year. ${ }^{1}$ Although many factors contribute to suicide, there is mounting evidence that exposure to suicide in some form can lead to imitative or "copycat" suicides. This concept of suicide "contagion" has existed for many years; however, it has generally received little empirical attention. Many aspects of this phenomenon remain mysterious, leaving us with limited ability to identify those at risk and suggest evidence-based postvention strategies. What we really want to know is, who is at risk and how can we help them?

Most research on suicide contagion has focused on natural experiments in which an initial suicide (often of a celebrity) leads to imitative suicidal behaviour or clusters of subsequent suicidal events, usually associated with insensitive or sensational media coverage. ${ }^{2,3}$ This focus on unusual suicide events and celebrity suicide reduces our confidence in the broader applicability of the findings of the research. In this sense, research on suicide contagion is in its infancy. What have been missing are prospective studies that examine the risk of contagion in association with pre-existing factors such as mental illness, stressful life events and socioeconomic status. Understanding the effect of these factors on the risk of contagion is critical to identifying target groups and time frames for effective postvention.

In this issue, Swanson and Colman ${ }^{4}$ report the findings from a nationally representative prospective cohort study, examining the association between exposure to suicide in youth and subsequent suicidality outcomes. This large, welldesigned study involving thousands of Canadian youth aged 12-17 years reports several important findings that may help us identify those most at risk of suicide contagion. In this study, youth who reported the suicide of a schoolmate were significantly more at risk than those with no exposure, with the effect most prominent in the youngest age group. For example, youth aged $12-13$ years who reported the suicide of a schoolmate were more likely to report suicidal ideation or attempt suicide (odds ratio 6.46 and 5.93, respectively, fully adjusted for sex, socioeconomic status and previous depression or anxiety), with residual risk present 2 years later. Importantly, and perhaps surprisingly, personally knowing the victim did not increase the risk of suicidality outcomes, and having social support, depression or anxiety did not affect the associations. However, having a previous stressful life event did increase the risk: students who had experienced 1 or more stressful life events were at a greater risk of suicidality outcomes.

What does this study tell us? It provides convincing evidence that, among young people, exposure to suicide is a risk factor for future suicidal behaviour. This is extremely important because it tells us that everyone who is exposed to suicide should be considered when postvention strategies are developed. The study also reveals that the youngest are most at risk, and that the risk is long-lasting. Many postvention strategies, which typically span the months after a suicide, may not be long enough to truly reduce the risk of contagion.

The results of this study challenges some widely held ideas. For example, individuals who knew the victim are typically seen to be at greatest risk of being affected by exposure; however, Swanson and Colman ${ }^{4}$ did not find evidence of this. Similarly, previous poor mental health did not modify the association between exposure and
Competing interests: None declared.

This article was solicited and has not been peer reviewed.

Correspondence to: India Bohanna, india.bohanna@jcu.edu.au

CMAJ 2013. DOI:10.1503 /cmaj.130678 
subsequent suicidality, although previous stressful life events did. This study tells us that all young people are at risk following the suicide of a schoolmate, not only those close to the victim or those with pre-existing mental health issues.

Like most good research, this study leaves us with as many questions as answers. Where do we go from here? Given the clear evidence of generalized risk of contagion shown by Swanson and Colman, ${ }^{4}$ several responses are urgently required. Several authors have noted the severe lack of suicide prevention and postvention research. ${ }^{5} \mathrm{~A}$ recent review by Cox and colleagues ${ }^{6}$ found few evaluated postvention strategies targeting suicide contagion in young people, suggesting that we know very little about what works to prevent contagion. In-depth studies are required that seek to understand the psychological and cognitive processes that occur in young people following exposure to suicide and how such processes lead to increased ideation and suicidal behaviour. A mechanistic understanding of how this risk is conferred is going to be critical to develop effective intervention strategies. Clearly, more well-designed and comprehensively evaluated prevention and postvention interventions are also urgently required.
The idea that suicide is contagious has always been controversial for various reasons; however, this important study should put many, if not all, doubts to rest. A unified and concerted effort now needs to be directed toward developing evidencebased postvention strategies. We need to know what works in mitigating the risk of contagion and why. Only then can such strategies be confidently used to help those most vulnerable to the aftereffects of suicide: our youth.

\section{References}

1. Suicide prevention (SUPRE). Geneva (Switzerland): World Health Organization; 2011. Available: www.who.int/mental _health/prevention/suicide/suicideprevent/en/index.html (accessed 2013 Apr. 15)

2. Niederkrotenthaler T, Fu KW, Yip PS, et al. Changes in suicide rates following media reports on celebrity suicide: a meta-analysis. J Epidemiol Community Health 2012;66:1037-42

3. Bohanna I, Wang X. Media guidelines for the responsible reporting of suicide; a review of effectiveness. Crisis 2012;33:190-8.

4. Swanson SA, Colman I. Association between exposure to suicide and suicidality outcomes in youth. CMAJ 2013;185:870-7.

5. Huisman A, Pirkis J, Robinson J. Intervention studies in suicide prevention research. Crisis 2010;31:281-4.

6. Cox GR, Robinson J, Williamson M, et al. Suicide clusters in young people: evidence for the effectiveness of postvention strategies. Crisis 2012;33:208-14.

Affiliation: India Bohanna is with the Department of Public Health, Tropical Medicine and Rehabilitation Science, James Cook University, Cairns, Queensland, Australia.

\section{GET THE CREDIT YOU DESERVE}

New online accredited

CME courses just released

Free for CMA members

cma.ca/cme-july
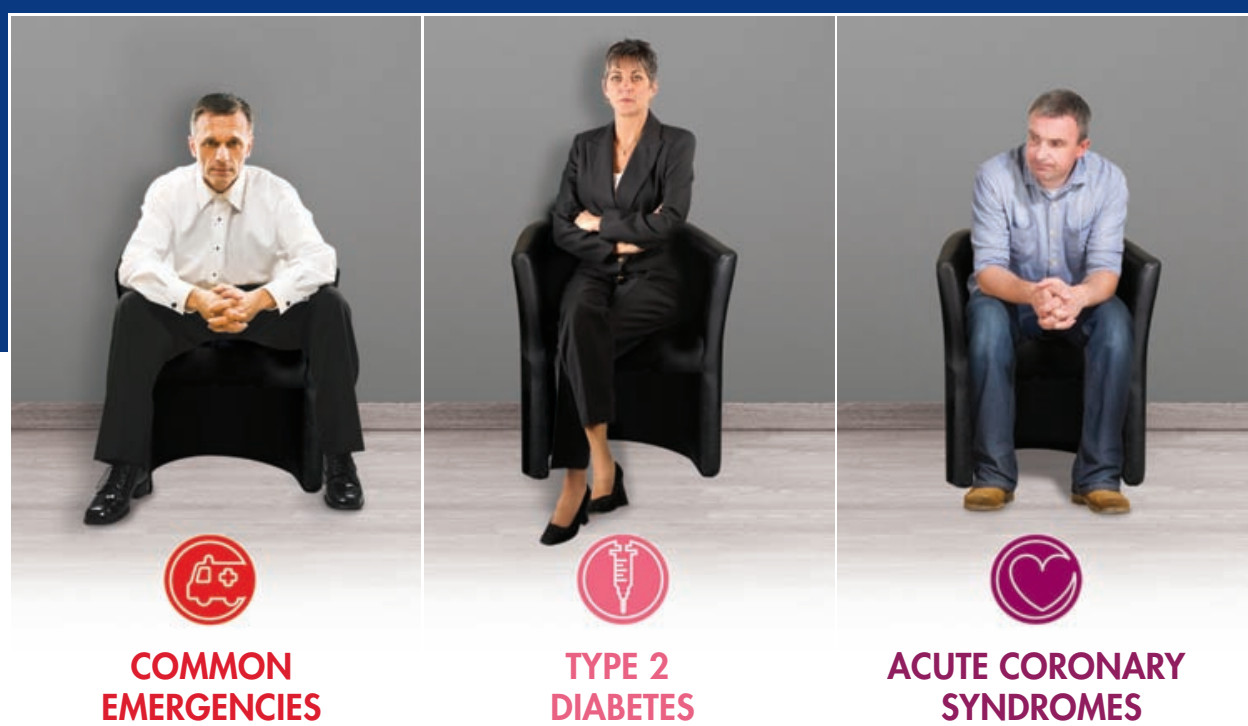

These courses were developed in collaboration with the CMA and Memorial University Professional Development and Conferencing Services through an educational grant from Pfizer Canado.
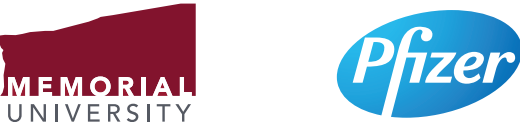

UNIVERSITY

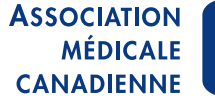

MÉDICALE
CANADIENNE
CANADIAN

MEDICAL

ASSOCIATION 